
allemande

46-2 | 2014

Intellectuels et politique en Allemagne

\title{
L'Allemagne et l'Euro : les enseignements de la balance des paiements allemande
}

\section{Béatrice Dedinger}

\section{(2) OpenEdition \\ 12 Journals}

Édition électronique

URL : https://journals.openedition.org/allemagne/1862

DOI : 10.4000/allemagne.1862

ISSN : 2605-7913

Éditeur

Société d'études allemandes

Édition imprimée

Date de publication : 30 décembre 2014

Pagination : 483-496

ISSN : 0035-0974

\section{Référence électronique}

Béatrice Dedinger, "L'Allemagne et l'Euro : les enseignements de la balance des paiements

allemande », Revue d'Allemagne et des pays de langue allemande [En ligne], 46-2 | 2014, mis en ligne le

29 juillet 2019, consulté le 18 mai 2021. URL : http://journals.openedition.org/allemagne/1862 ; DOI

https://doi.org/10.4000/allemagne.1862 


\section{L'Allemagne et l'Euro: les enseignements de la balance des paiements allemande}

\section{- Béatrice Dedinger*}

L'excédent commercial de l'Allemagne est connu du monde entier. C'est assez remarquable, car qui connaît, même en Europe, le solde de la balance commerciale britannique, italienne, belge ou polonaise? Il est vrai que le solde allemand a atteint une ampleur sans précédent. En 2013, il est de 198 milliards d'euros, soit 7,2 \% du PIB. Ce résultat reste exceptionnel lorsqu'on le compare à celui d'autres pays. L'excédent de la Chine est de 188 milliards d'euros, soit moins de $3 \%$ de son PIB. Les Pays-Bas ont un solde commercial positif élevé en pourcentage du PIB $(7,1)$ mais bien moindre que celui de l'Allemagne en valeur (44 milliards d'euros) ${ }^{(1)}$. De nombreuses critiques sont adressées à cet excédent allemand jugé trop élevé, encouragé par des mesures politiques et générateur de risques pour les pays partenaires: « [Germany's] current-account surplus, at $7 \%$ of GDP, is now more than three times higher than it was a decade ago, largely thanks to an artificially cheap currency and squeezed wages. It is unhealthy, both for Germans (who forgo higher living standards to pile up savings that are poorly invested abroad) and for others in the euro zone and beyond ${ }^{(2)}$. Or les mêmes critiques étaient déjà formulées il y a quarante ans. En effet, l'excédent est apparu en 1952 et depuis, jamais la balance commerciale allemande n’a été déficitaire. Les économistes se sont très vite intéressés à ce phénomène et l'ont analysé dans les mêmes termes quaujourd'hui: "the chronic surpluses meant that other countries were continuously buying more goods and services from the Federal Republic than they were delivering to her. The question which both Germany and the international community sooner or later had to face up was this: how long could the export surpluses continue without impoverishing Germany in real terms and bankrupting the other nations of their means of payment? $»^{(3)}$.

* Chercheur au Centre d'histoire de Sciences Po Paris.

1 Résultats 2013. Source: site internet du FMI.

2 The Economist, "Europe's reluctant hegemon », 15 juin 2013, p. 14.

3 Patrick M. Boarman, Germany's economic dilemma. Inflation and the balance of payments, New Haven/Londres, Yale University Press, 1964, p. x. 
Faut-il en conclure que rien n'a changé depuis plus d'un demi-siècle et que la seule question à se poser au sujet du solde commercial allemand porte sur sa longévité persistante? Le graphique 1 ci-dessous apporte une réponse claire. Il met en évidence un changement spectaculaire dans l'évolution du solde de la balance commerciale allemande depuis 2000, qui affecte également les balances courante et financière. L'objet de cet article est d'expliquer le graphique 1. Il propose une étude sur le long terme de la balance des paiements de l'Allemagne qui, à ma connaissance, n'a pas encore été faite ${ }^{(4)}$. Cette étude vise à remettre en question l'analyse traditionnelle de l'excédent commercial allemand en montrant que depuis 2000, soit depuis la création de l'Union monétaire européenne, de nouveaux mécanismes sont entrés en jeu dont on peut mesurer l'impact en regardant l'évolution de la balance des paiements allemande. Pour mieux comprendre ces mécanismes, quelques notions de base sur le fonctionnement de la balance des paiements doivent tout d'abord être rappelées au lecteur.

Graphique 1: Les soldes de la balance des paiements de l'Allemagne, 1950-2013 (millions d'euros)

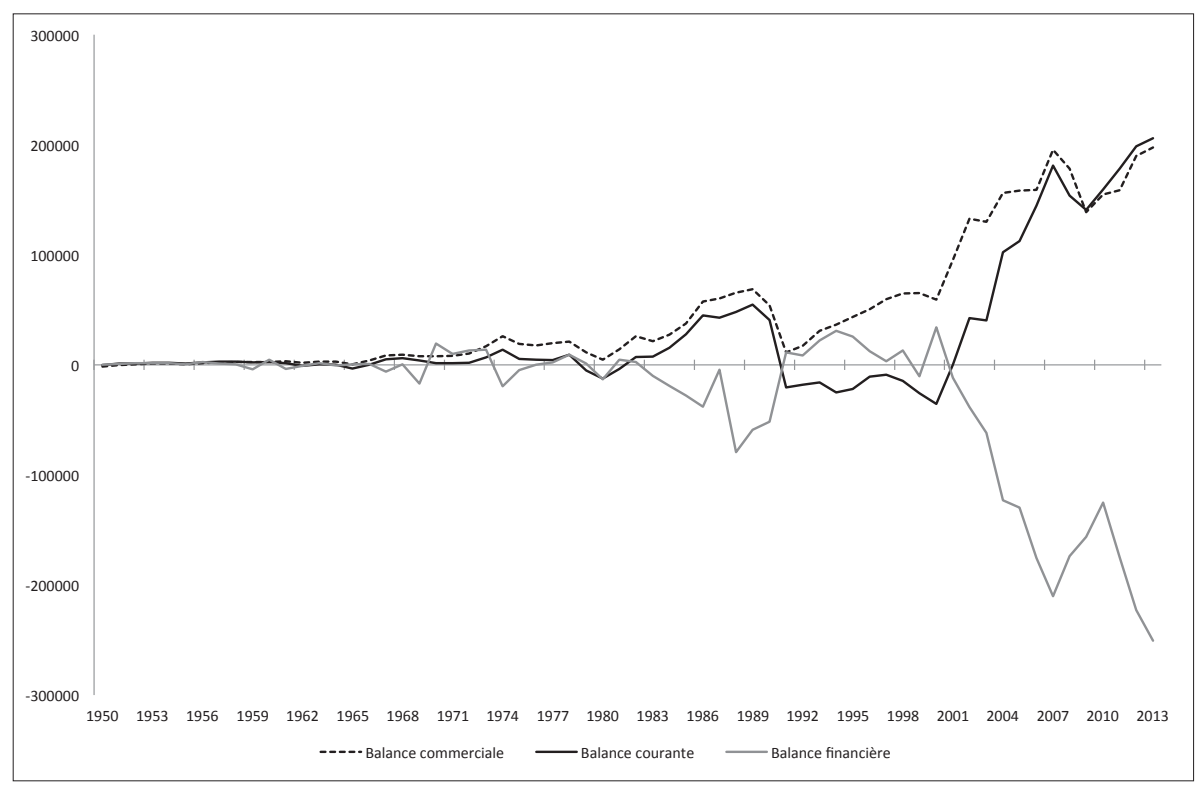

Source: Deutsche Bundesbank, Zahlungsbilanzstatistik. Statistisches Beiheft 3 zum Monatsbericht (plusieurs années).

\section{Quelques notions de base sur la balance des paiements}

La balance des paiements d'un pays est un document statistique élaboré sous forme comptable qui enregistre toutes les opérations que les résidents effectuent avec les non-

4 On trouve une analyse de la balance des paiements allemande sur la période 1950-1990 dans Herbert Giersch, Karl-Heinz Paqué, Holger Schmieding, The fading miracle. Four decades of market economy in Germany, Cambridge University Press, 1992, p. 236-250. 
résidents au cours d'une année ${ }^{(5)}$. C'est une balance équilibrée, chaque transaction, financière ou non financière, avec un non-résident, inscrite au crédit ou au débit de la balance, a une contrepartie financière, c'est-à-dire une variation d'avoirs ou d'engagements vis-à-vis des non-résidents. Dans le cas le plus simple, une exportation de marchandises ou une cession de titres par les résidents donne naissance en contrepartie à une augmentation de leurs avoirs liquides (par exemple, les dépôts des résidents auprès des banques non résidentes) ou à une diminution de leurs engagements (par exemple un remboursement d'avances précédemment obtenues auprès de banques non résidentes). À la vision verticale, en deux parties, de la balance des paiements correspond une vision horizontale, en deux parties également ${ }^{(6)}$. Au-dessus de la ligne sont enregistrées les opérations de nature réelle: échanges de marchandises, échanges de services (tourisme, transport, assurance...), échanges de revenus (salaires du travail, intérêts des capitaux), transferts unilatéraux (dons ou paiements versés à ou reçus de l'étranger). La différence entre le total des opérations réelles inscrites au crédit et celles inscrites au débit est égale au solde de la balance courante. La balance commerciale est une sous-balance de la balance courante calculée en soustrayant les importations (débit) des exportations de marchandises (crédit). L'ensemble des opérations inscrites au-dessus de la ligne est compensé par des opérations de nature financière qui sont enregistrées en dessous de cette ligne. On inscrit au crédit les entrées ou importations de capitaux (dettes vis-à-vis de l'étranger) et au débit, les sorties ou exportations de capitaux (créances sur l'étranger). Un excédent de la balance financière correspond donc à un endettement net du pays vis-à-vis de l'extérieur. Les postes du compte financier incluent: les investissements directs (contrôle ou influence significative sur l'entreprise, investissement durable), les investissements de portefeuille (moindre influence sur l'entreprise, capitaux volatiles, liquides, flexibles), les produits dérivés et stock-options (nouvelle rubrique du compte financier créée en 2000, s'apparente plus à un transfert de risque qu'à une offre de fonds), les autres investissements (poste résiduel qui englobe toutes les opérations sur actifs et passifs financiers vis-à-vis des non-résidents qui ne figurent pas sous une autre rubrique du compte financier; crédits commerciaux, prêts et emprunts, autres avoirs et engagements), et les réserves officielles (avoirs extérieurs détenus par les autorités monétaires). Étant donné le principe de comptabilité en partie double, les soldes du compte financier et du compte courant se compensent; le solde de la balance des paiements proprement dite est toujours égal à zéro.

Un excédent (déficit) de la balance courante est exactement compensé par un déficit (excédent) de la balance financière. Cette égalité comptable dans les opérations d'un pays avec l'extérieur a une contrepartie au niveau de l'économie domestique. À un excédent de la balance courante correspond toujours, ex post, un excédent de l'épargne domestique sur l'investissement domestique. En effet, les résidents d'un pays peuvent utiliser leur revenu (après impôt) soit pour consommer la production domestique, soit sous forme d'épargne investie dans l'économie domestique, soit sous forme d'épargne

5 Les définitions en usage aujourd'hui sont celles du International Monetary Fund, Balance of Payments and International Investment Position, Sixth Edition (BPM6), IMF, Washington DC, 2009, 351 p.

6 Le $5^{\mathrm{e}}$ manuel de la balance des paiements du FMI introduit un troisième compte, le compte de capital, qui regroupe les transferts en capital et les acquisitions et cessions d'actifs non financiers non produits (brevets, marques, droits d'auteur...). Pour simplifier, on ne considère ici que les balances courante et financière. 
investie à l'étranger. Quand une part est investie à l'étranger, c'est qu'elle n'est pas disponible pour la consommation ou l'investissement domestique. Un surplus d'épargne domestique correspond donc à la fois à une exportation de capital vers l'étranger et à une exportation des biens non consommés dans le pays. C'est l'inverse qui se produit pour un pays ayant un déficit de la balance courante: il connaît un déficit d'épargne domestique, les résidents consomment ou investissent plus qu'ils ne produisent. Ce déficit d'épargne est couvert par une importation de capital étranger égale au surplus d'importations de marchandises pour couvrir la consommation domestique. Un excédent commercial ne doit donc pas être vu comme un signe d'enrichissement du pays. Il n'a pas nécessairement pour contrepartie une augmentation des entrées de devises, ce peut être aussi une augmentation des exportations de capitaux qui vont s'investir à l'étranger au lieu de financer la croissance domestique.

Que se passe-t-il lorsque la balance des paiements d'un pays est en déséquilibre? On sous-entend en général un déséquilibre de la balance courante. Des mécanismes de retour à l'équilibre entrent en action, qui sont différents selon que le pays est en changes flexibles, fixes ou dans une union monétaire. Supposons qu'un pays enregistre un déficit commercial. Cette situation induit un excédent d'achats de marchandises étrangères qui a pour contrepartie une demande excédentaire de devises étrangères contre monnaie domestique. La monnaie domestique a donc tendance à se déprécier. Dans un système de changes flexibles, les autorités monétaires ne sont pas contraintes d'intervenir pour maintenir la parité de la monnaie. Elles peuvent la laisser se déprécier, ce qui réduit le prix des biens domestiques en monnaie étrangère et relance les exportations du pays, sans que la situation monétaire intérieure ait été affectée. Cependant, si le taux d'intérêt est relativement élevé, que les capitaux étrangers sont attirés dans le pays et entretiennent un excédent de la balance financière, le retour à l'équilibre commercial peut ne pas se produire. Un déséquilibre durable peut s'instaurer qui a des conséquences sur le change de la monnaie. Mais les autorités monétaires du pays ne sont pas tenues d'intervenir en changes flottants. Dans un système de changes fixes, elles ont cette obligation. Si elles veulent éviter une dévaluation et maintenir la parité fixée, elles vont vendre des devises étrangères (baisse des réserves officielles, augmentation du solde débiteur de la balance financière) contre de la monnaie domestique. Ce faisant, l'offre de monnaie domestique dans le pays se raréfie, d'où une tendance à la baisse des prix domestiques qui stimule les exportations du pays et ramène le solde commercial à l'équilibre. Raréfier l'offre de monnaie domestique afin de réduire les importations peut aussi passer par une augmentation du taux d'intérêt. Cette mesure peut avoir pour effet d'attirer les capitaux étrangers qui viennent gonfler l'offre de monnaie domestique et contrer l'objectif initial de maintien de la parité monétaire. Un recours au contrôle des mouvements de capitaux permet de conserver l'autonomie de la politique monétaire dans un système de changes fixes. Enfin, si le pays est membre d'une union monétaire, il perd le contrôle de la politique de change et de la politique monétaire puisque celui-ci est délégué à la banque centrale de l'union. Dans ce cas, un déficit commercial ne peut être résorbé ni par une vente de devises, ni par une dépréciation de la monnaie, ni par une mesure de politique monétaire. La relative faiblesse de la demande de produits domestiques tend à faire baisser les prix de ces produits et des facteurs de production concernés, ce qui peut relancer les exportations. Si les prix et les salaires ne baissent pas, l'ajustement se fera par les quantités, c'est-à-dire par une baisse de la production et une baisse de l'emploi, ce que l'on appelle aussi une crise économique. 


\section{L'explication traditionnelle de l'excédent commercial allemand}

Contrairement à ce que décrit le mécanisme de retour à l'équilibre décrit ci-dessus, l'excédent de la balance commerciale allemande n'a pas disparu depuis 1952. Pour être précis, ce qui est le plus surprenant, c'est l'excédent quasi-permanent de la balance courante. Celui-ci est dû principalement à l'excédent de la balance commerciale, c'est pourquoi les deux sont souvent confondus. Mais il faut bien voir que, si l'excédent commercial était compensé par un déficit équivalent de la balance des services par exemple (les Allemands iraient dépenser en voyages à l'étranger les devises gagnées sur les exportations), la balance commerciale allemande ne susciterait pas autant d'émoi. La question des causes de l'excédent commercial persistant a été posée dans les années $1960^{(7)}$. Comme l'a écrit un grand historien économiste, "I have for years been expecting the German surplus to go away. Perhaps an attempt to "explain" this surplus would exorcise it in medieval fashion ${ }^{\left({ }^{(8)}\right.}$. Les explications qu'il a données à cette époque seront revues dix ans plus tard sans grandes modifications ${ }^{(9)}$. Ce sont toujours les mêmes qui sont retenues au début du $\mathrm{XXI}^{\mathrm{e}}$ siècle. Il peut y avoir une certaine cohérence dans le fait qu'un excédent permanent soit expliqué par des causes permanentes mais, comme nous le verrons, une nouvelle explication doit être trouvée pour comprendre la croissance de l'excédent commercial depuis une dizaine d'années. Quatre types de causes sont traditionnellement retenus: une forte propension de l'Allemand à exporter, une structure des exportations avantageuse et des prix compétitifs sont à l'origine de la force des exportations allemandes; la forte propension à épargner des Allemands explique la faiblesse des importations.

La réputation du commerçant allemand ne date pas de la seconde moitié du $\mathrm{xx}^{\mathrm{e}}$ siècle, elle remonte loin dans l'histoire, au moins à l'âge d'or des villes hanséatiques aux $\mathrm{XIV}^{\mathrm{e}}-\mathrm{XV}^{\mathrm{e}}$ siècles. C'est comme si la "fibre " ou le " gène " du commerce étaient inscrits dans la psyché ou la mémoire cellulaire du peuple allemand. "L'idée même de commerce comme source de prospérité apparaît en Allemagne avec une intensité et un caractère "existentiel” qu'elle ne présente dans aucun autre pays, à l'exception du Japon ${ }^{(10)}$. L'histoire tourmentée de l'entre-deux-guerres a accentué ce trait. Tout d'abord, en coupant le commerçant allemand de l'accès aux marchés étrangers durant vingt ans ${ }^{(11)}$; d'autre part, elle a créé un sentiment d'insécurité collective à laquelle le renforcement des liens commerciaux et de l'interdépendance économique avec les autres pays permet de répondre ${ }^{(12)}$. Mais il ne suffit pas d'avoir la volonté d'exporter, il faut encore que les produits que l'on fabrique soient demandés par l'étranger. On

7 P. M. Boarman, Germany's economic dilemma (note 3); Charles P. Kindleberger, "Germany's persistent balance-of-payments disequilibrium ", in: Robert E. BALDwin et al., Trade, growth, and the balance of payments: essays in honor of Gottfried Haberler, Chicago, Rand McNally, 1965, p. 230-248; Joseph Markus, "Some observations on the West German trade surplus ", Oxford Economic Papers, 17/1, mars 1965, p. 136-145.

8 C. P. Kindleberger, « Germany’s persistent balance-of-payments disequilibrium » (note 7), p. 230.

9 Charles P. Kindleberger, Germany's persistent balance-of-payments disequilibrium revisited, German Studies Notes, Indiana, Institute of German Studies, 1976, 58 p.

10 Wolfgang Hager, «L'Allemagne, un commerçant hors du commun », Politique étrangère, 1 (1979), p. 35.

11 C. P. Kindleberger, Germany's persistent balance-of-payments disequilibrium revisited (note 9), p. 20.

12 Cet argument est si souvent évoqué qu'il n'est plus besoin de référence. La sécurité commerciale est un moyen de pallier la sécurité militaire. Cela rejoint aussi l'idée de paix par le commerce. 
insiste beaucoup depuis l'après-guerre sur la « chance » des entreprises allemandes de produire précisément les biens qui sont l'objet d'une forte demande internationale ${ }^{(13)}$ et dont l'élasticité-prix est faible. Sur ce point, je renvoie à un article paru dans cette revue, dans lequel il est montré que la spécialisation allemande actuelle relève d'un long processus historique. Ce n'est pas simplement par « chance » que les entreprises allemandes ont aujourd'hui des avantages comparatifs élevés dans les véhicules, les machines, les produits chimiques et les appareils électriques ${ }^{(14)}$.

La troisième catégorie de causes touche à l'aspect le plus controversé de l'excédent. Si les produits allemands se vendent bien, c'est parce que leur prix est relativement peu élevé. Un grand nombre de facteurs peuvent avoir une incidence sur ce prix, parmi lesquels on retient: la politique de change, la politique monétaire et les coûts salariaux. Ils sont pour la plupart de nature politique, ce qui explique qu'ils soient le plus soumis aux critiques. Dans les années 1950-60, on a beaucoup reproché à l'Allemagne la sousévaluation du deutschemark, bien que la monnaie allemande ait été réévaluée à plusieurs reprises avant l'effondrement du système de Bretton Woods en 1973 et le passage à un système de changes flottants ${ }^{(15)}$. À partir de là, les forces du marché pousseront à l'appréciation du deutschemark, et c'est la politique monétaire qui devient la cible des critiques. La politique anti-inflationniste poursuivie par la Bundesbank accroît le différentiel de prix avec les pays inflationnistes et favorise les exportations ${ }^{(16)}$. La pression internationale poussera l'Allemagne à adopter une politique monétaire plus expansionniste, mais celle-ci prendra fin avec le nouveau choc pétrolier ${ }^{(17)}$. La réunification allemande provoque une baisse soudaine de l'excédent commercial et l'apparition d'un déficit de la balance courante qui se maintiendra jusqu'en 2000. Quand le problème de l'excédent allemand revient sur le devant de la scène, la politique monétaire est devenue commune. Ce sont maintenant les coûts salariaux que l'on rend responsables de la compétitivité des prix allemands. Les réformes mises en place à partir de 2003 par le gouvernement Schröder visaient à relancer la compétitivité allemande notamment par une libéralisation du marché du travail qui s'est traduite par l'apparition d'emplois à bas salaires. Il faut toutefois souligner que la relative faiblesse des coûts salariaux était déjà citée comme une des causes de l'excédent dans les premières années d'aprèsguerre et qu'on l'attribuait à la retenue des travailleurs allemands ${ }^{(18)}$.

Peut-on dire que l'excédent commercial permanent de l'Allemagne depuis 1952 est le résultat d'une politique délibérée du gouvernement allemand comme on l'en accuse

13 Les entreprises allemandes productrices de biens d'équipement ont eu la chance de profiter du boom de la guerre de Corée. On retrouve le même argument cinquante ans plus tard: «German firms were also lucky to be making the right stuff at the right time ", in: The Economist, "Europe's reluctant hegemon », 15 juin 2013, p. 12.

14 Cf. Béatrice Dedinger, "L'avenir commercial de l'Allemagne ", Revue d'Allemagne et des pays de langue allemande, t. 45, janvier-juin 2013, p. 107-120.

15 W. HAGER, «L'Allemagne, un commerçant hors du commun » (note 10), p. 39; C. P. KindLEBERGER, Germany's persistent balance-of-payments disequilibrium revisited (note 9), p. 40. Giersch/Paqué/Schmieding, The fading miracle (note 4), p. 240-243.

18 Henry C. Wallich, Mainsprings of the German revival, New Haven, Yale University Press, 1955, p. 297-304; C. P. Kindleberger, "Germany's persistent balance-of-payments disequilibrium » (note 7), p. 235-237. 
fréquemment ${ }^{(19)}$ ? La réponse est ambiguë. L’objectif permanent de la politique économique allemande depuis la création de la RFA est la stabilité des prix. La réalisation de cet objectif a aussi permis le maintien d'une monnaie forte. Ces deux données ont des effets contrastés sur les échanges extérieurs: la force de la monnaie n'est pas favorable aux exportations, mais elle permet de réduire la valeur des importations en monnaie domestique (d'où la moindre incidence qu'a eue l'augmentation des prix du pétrole sur la balance commerciale allemande); la stabilité des prix est favorable aux exportations. Si l'on ne peut affirmer que la politique économique de l'Allemagne a eu pour objectif l'obtention d'un excédent commercial/courant, il est en revanche certain que le gouvernement allemand n'a pas eu de politique délibérée de réduction de l'excédent ${ }^{(20)}$ (sauf sous la pression internationale dans les années 1970). Pour les Allemands, l'excédent commercial est un signe de bonne santé de leur économie qui ne nécessite pas de traitement correcteur ${ }^{(21)}$.

L'autre versant de l'excédent commercial est la relative faiblesse des importations. Celle-ci est attribuée à la forte propension à épargner des Allemands. Le lien entre commerce extérieur et épargne découle directement de l'égalité comptable, rappelée dans les notions de base, entre l'excédent de la balance commerciale/courante et l'excédent de l'épargne domestique sur l'investissement domestique. Un excédent de la balance courante se traduit nécessairement par des exportations nettes de capitaux vers l'étranger qui correspondent à de l'épargne non investie dans le pays de résidence. Cet excédent d'épargne peut provenir des ménages, des entreprises ou de l'État. Ainsi, dans les premières années de la RFA, l'excédent d'épargne est venu du gouvernement allemand qui, par une politique de forte taxation, a généré un surplus d'exportations ${ }^{(22)}$. Depuis une dizaine d'années, on observe une augmentation du taux d'épargne nationale allemand alors qu'il tend à baisser dans les autres pays. Comme cette hausse ne vient pas des ménages, on peut l'attribuer aux entreprises allemandes. D'où l'une des principales explications de l'excédent commercial allemand aujourd'hui: «Si le partage des revenus avait été plus favorable aux salariés en Allemagne ou si les entreprises allemandes avaient investi une partie plus importante de leurs profits, l'excédent extérieur de l'Allemagne ne serait pas apparu. La zone euro globalement aurait eu un déficit extérieur (la demande intérieure aurait été plus forte en Allemagne) et, à l'équilibre, des taux d'intérêt plus élevés jusqu'à la crise. Il aurait été plus difficile pour les pays périphériques de refinancer auprès de l'Allemagne l'en-

19 Les critiques ont commencé dès les années 1950. Cf. Egon SoHmen, "Competition and growth: the lesson of West Germany », The American Economic Review, 49/5, décembre 1959, p. 995.

20 Voir l'étude de Michael Michaely, Balance of Payments Adjustment Policies: Japan, Germany, and the Netherlands, NBER, 1968, p. 58-84. Elle porte sur une dizaine d'années d'après-guerre et montre que le réajustement de la balance des paiements n'est pas un objectif de la politique des autorités allemandes (gouvernement et banque centrale).

21 "In German eyes, strong exports and a big trade surplus are symbols of virility », in: The Economist (note 13), p. 12.

22 Cf. C. P. Kindleberger, « Germany's persistent balance-of-payments disequilibrium » (note 7), p. 240. Les réserves de changes accumulées devaient servir à payer la contribution de l'Allemagne à l'effort de défense européenne. Elles sont appelées « Julius Turm » à l'image du trésor de guerre mis de côté en 1871 sur l'indemnité reçue des Français. 
dettement du secteur privé, et la bulle de l'endettement et de l'immobilier dans ces pays aurait été découragée. Sans cette bulle, la croissance de ces pays aurait été plus faible, mais celle de l'Allemagne plus forte, jusqu'à la crise, avec le supplément de demande intérieure. Aujourd'hui, les excédents d'épargne de l'Allemagne financent le soutien des banques et des États des pays périphériques après la crise. Mais, avant la crise, une politique différente de partage des revenus en Allemagne conduisant à un moindre excédent extérieur aurait été stabilisante en évitant de financer les bulles spéculatives des pays périphériques " ${ }^{(23)}$.

De nos jours, deux causes principales sont donc retenues pour expliquer l'excédent commercial allemand: la compétitivité-prix, due à la faiblesse des coûts salariaux, et la propension à épargner des Allemands. Les prix des produits allemands sont compétitifs parce que les salariés allemands ont accepté une baisse de leur salaire. Cette baisse de salaire induit une baisse de la consommation. Et comme par ailleurs, les entreprises épargnent trop et n'investissent pas assez, l'Allemagne a un surplus de biens et d'épargne à exporter. Cette épargne excédentaire pèse sur les taux d'intérêt de la zone euro, incitant les pays périphériques, plus inflationnistes et plus dépensiers que l'Allemagne, à s'endetter excessivement. Il y a certainement une part de vérité dans cette analyse mais, comme nous allons le voir, elle manque de recul historique et n'explique pas la rupture de 2000 .

\section{La création de l'Union monétaire européenne et ses conséquences sur la balance des paiements allemande}

Cela fait soixante ans que l'on reproche aux Allemands leurs prix «trop » bas et leur épargne « trop » forte. Il est difficile de croire que ces deux facteurs aient subi des changements d'une ampleur telle qu'ils aient provoqué l'accroissement soudain et massif de l'excédent commercial/courant allemand à partir de 2000. Revenons à la définition d'un excédent courant et aux notions de base de la balance des paiements. Un excédent courant correspond: à une exportation de biens et services supérieure à l'importation de biens et services; à une épargne domestique supérieure à l'investissement; à des exportations de capitaux supérieures aux importations de capitaux. Une augmentation de l'excédent courant peut donc provenir de plusieurs sources: augmentation des exportations de biens et services, baisse des importations de biens et services, augmentation de l'épargne domestique, baisse de l'investissement domestique, augmentation des exportations de capitaux, baisse des importations de capitaux (ou augmentation des « désimportations » de capitaux = retrait de capitaux étrangers placés dans le pays, ce qui s'apparente à une exportation de capital). Seules les deux dernières sources sont susceptibles de connaître de soudaines variations à court terme. Cela nous conduit à la balance financière allemande.

Le graphique 1 montre que, symétriquement à la balance courante, la balance financière se retourne à partir de 2000 pour devenir fortement et durablement négative. L'Allemagne exporte donc beaucoup plus de capitaux qu'elle n'en importe depuis 2000.

23 Patrick Artus, «L'excédent extérieur de l'Allemagne est-il légitime? Est-il dangereux? », Natixis Flash Économie, $\mathrm{n}^{\circ} 476,6$ juillet 2012, p. 5-8. 
Les statistiques allemandes de la balance des paiements permettent de décomposer ces capitaux par catégorie d'investissements, par agent économique et par pays. Trois phénomènes majeurs se dégagent des graphiques 2 à 4 ci-dessous: 1 . Le retournement du déficit financier à partir de 2000 est dû à la catégorie " autres investissements » qui inclut les crédits commerciaux, les prêts et autres avoirs (engagements) non répertoriés dans les autres catégories de flux financiers; 2. Jusqu'en 2006, ce sont les institutions financières qui sont les principales exportatrices nettes de capitaux. La Bundesbank prend ensuite le relais; 3 . L'accroissement du déficit provient d'une augmentation massive des exportations nettes de capitaux vers les pays de la zone euro. Si l'on décompose par pays, un résultat très inattendu apparaît: cette augmentation massive résulte en majeure partie de retraits de capitaux belges et luxembourgeois d'Allemagne depuis $2000^{(24)}$. Tous ces éléments permettent de proposer une analyse de l'excédent commercial allemand fort différente de celle que l'on donne depuis quelques années ${ }^{(25)}$.

Graphique 2: Les soldes de la balance financière allemande par catégorie d'investissements, 1989-2013 (millions d'euros)

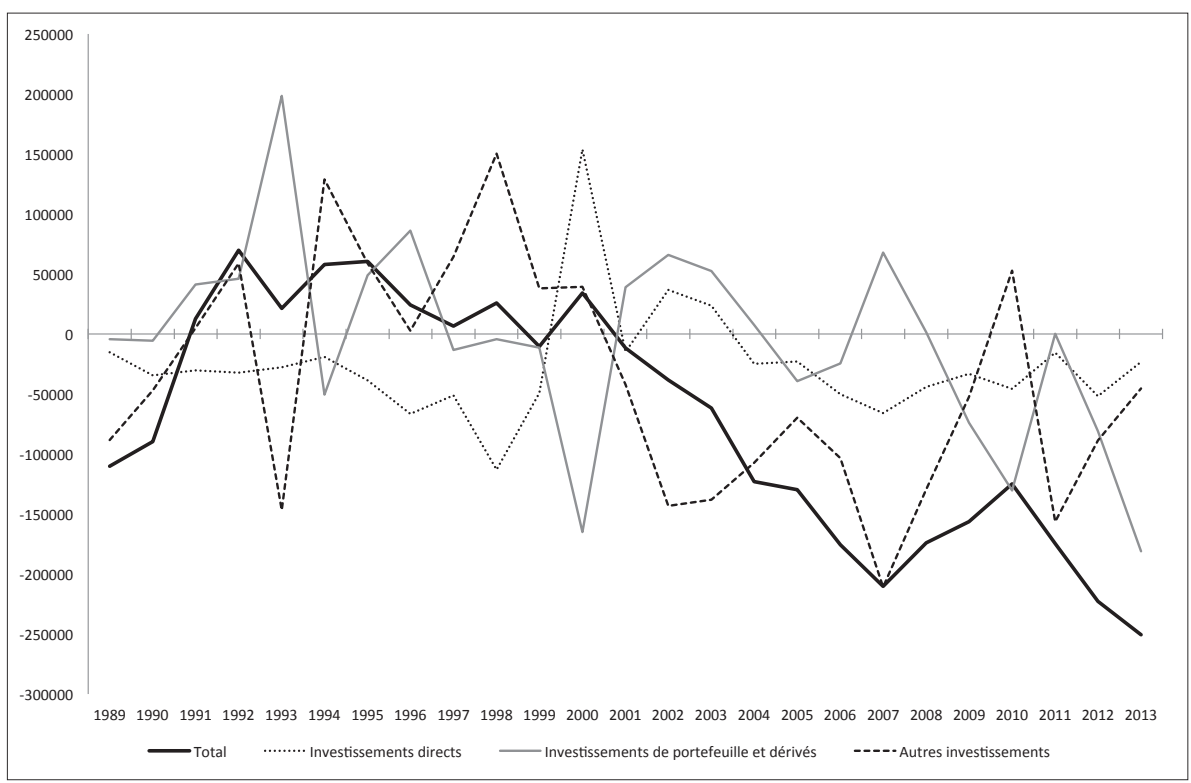

Source: Deutsche Bundesbank, Zahlungsbilanzstatistik. Statistisches Beiheft 3 zum Monatsbericht (plusieurs années).

24 Notons que la ventilation géographique des flux de capitaux est effectuée en fonction du pays de résidence de l'émetteur du titre pour les avoirs, et en fonction du pays de résidence de la première contrepartie non résidente connue pour les engagements.

25 Cette analyse rejoint celle de Hans-Werner Sinn, président de l'Institut für Wirtschaftsforschung (Ifo, Munich), dont la position hétérodoxe est très critiquée en Allemagne comme dans les autres pays de la zone euro. 
Graphique 3: Les soldes de la balance financière allemande par agent économique, 1999-2013 (millions d'euros)

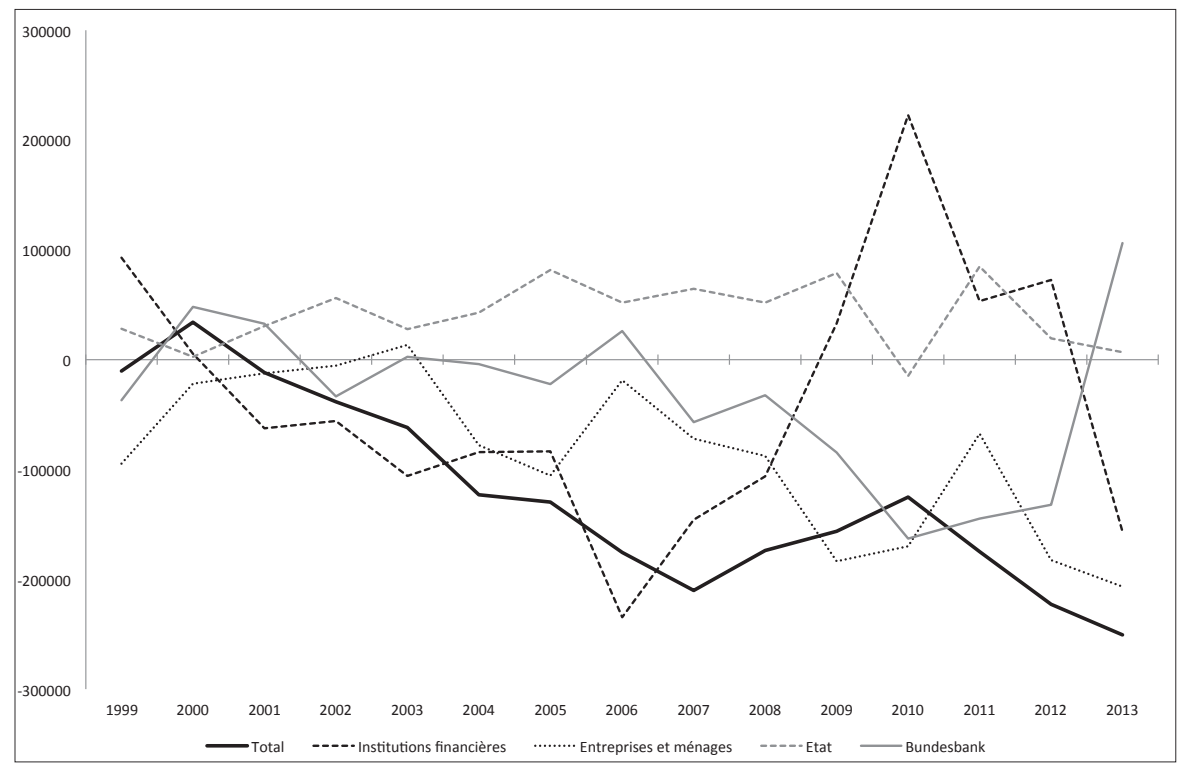

Source: Deutsche Bundesbank, Zahlungsbilanzstatistik. Statistisches Beiheft 3 zum Monatsbericht (plusieurs années).

Graphique 4: Les soldes de la balance financière allemande par pays, 1997-2013 (millions d'euros)

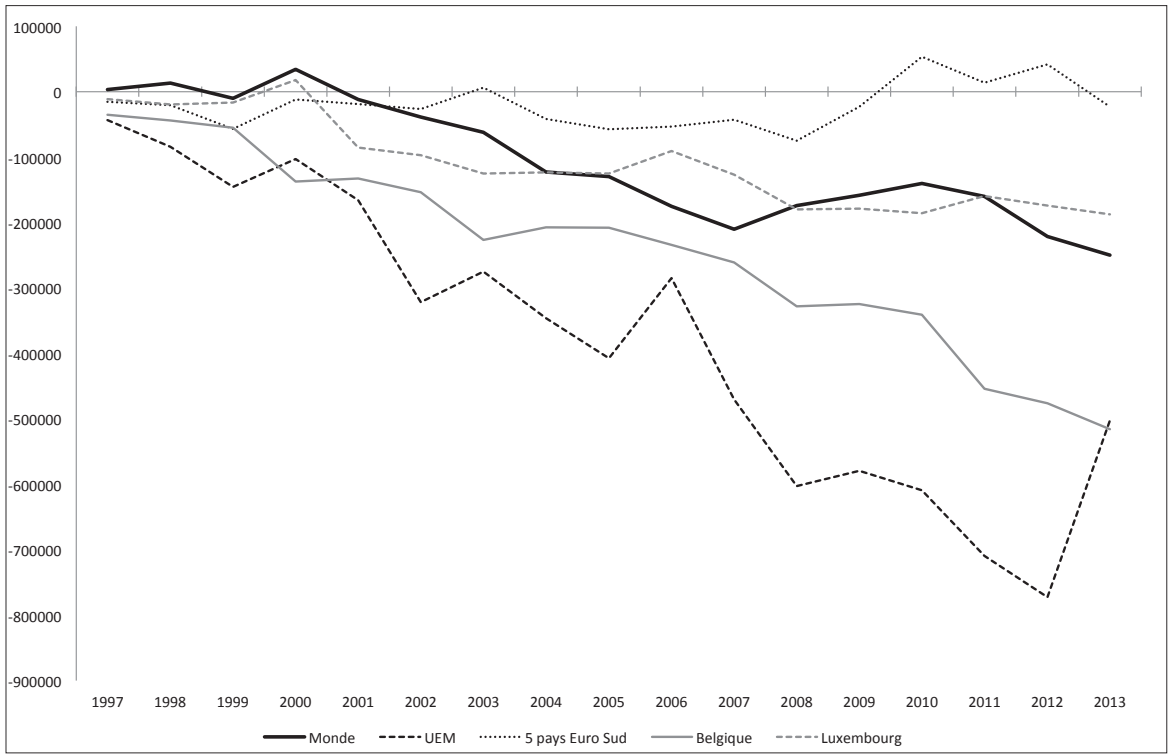

Source: Deutsche Bundesbank, Zahlungsbilanzstatistik. Statistisches Beiheft 3 zum Monatsbericht (plusieurs années). 
L'histoire commence avec la création de l'Union monétaire européenne. Le projet d'union monétaire a été relancé par le traité sur l'Union européenne signé en 1992 (traité de Maastricht). Celui-ci prévoit l'établissement d'une Union économique et monétaire (UEM), qui passe par l'instauration d'une monnaie unique et la définition d'une politique monétaire et d'une politique de change uniques. Le traité crée les institutions nécessaires à la mise en œuvre de cette union, Banque centrale européenne (BCE) et Système européen de banques centrales (SEBC). Il précise également les étapes de la réalisation de l'UEM. La deuxième étape débute le ler janvier 1994 avec la libéralisation totale des mouvements de capitaux entre les États membres, et entre États membres et pays tiers. C'est une phase transitoire avant la troisième étape qui démarre le $1^{\text {er }}$ janvier 1999 avec la création effective de l'UEM et de la monnaie unique. La libre circulation des capitaux et l'instauration d'une monnaie unique ont créé des conditions favorables au développement des échanges de capitaux, en particulier dans la zone euro. C'était d'ailleurs un des objectifs de la création de l'UEM.

La suppression des monnaies, donc des différentiels de change, des pays de l'UEM a fait disparaître la prime de risque que devaient payer les pays à monnaie faible pour attirer les capitaux étrangers. Des marchés de capitaux se sont développés dans ces pays, essentiellement l'Espagne, le Portugal et la Grèce. Ils sont devenus des importateurs nets de capitaux, et l'Allemagne, l'Autriche et les Pays-Bas ont été les plus gros exportateurs nets de capitaux de la zone euro. Ceci, soulignons-le à nouveau, s'est produit dans des proportions gigantesques ${ }^{(26)}$. Les capitaux exportés d'Allemagne sont, d'après les statistiques de la Bundesbank (graphique 4), en grande partie des capitaux belges sortis pour se placer sur d'autres marchés financiers ${ }^{(27)}$. Ces capitaux - des crédits bancaires et non plus des investissements directs ou de portefeuille - se seraient d'abord portés vers les banques françaises pour ensuite être exportés vers les pays du sud de la zone euro ${ }^{(28)}$. Ils ont servi à financer des investissements dans l'immobilier, qui ont eux-mêmes généré des revenus, lesquels ont stimulé la demande et les importations, ce qui a conduit aux importants déficits de la balance courante apparus à partir de la fin des années 1990. Ces déficits ont culminé en 2008 et ont été un facteur déclencheur de la crise qui a secoué toute l'Europe ${ }^{(29)}$.

Cette crise est la manifestation d'une réaction des investisseurs face au risque croissant que présentaient les pays endettés. Elle s'est traduite par un retrait des capitaux privés

26 Sur le développement des marchés financiers européens après la création de l'euro, voir European Central Bank, The monetary policy of the ECB, 2011, p. 43-48.

27 Sur cette question des capitaux belges, on notera que « le patrimoine financier net des particuliers belges, exprimé en pourcentage du PIB, est le plus élevé (203\%) des États membres de la zone euro pour lesquels ces données sont disponibles. Cette situation résulte de la constitution de considérables créances sur l'étranger, découlant de l'accumulation au fil du temps d'excédents sur le compte des opérations courantes avec le reste du monde ", in: Banque nationale de Belgique, Rapport 2012. Développements économiques et financiers, p. 105 (http://www.nbb.be/doc/ts/Publications/NBBreport/2012/ FR/T1/rapport2012_complete.pdf).

28 D'après Hans-Werner Sinn, Rescuing Europe, CESifo Forum, 11, août 2010, les banques françaises étaient très exposées sur les pays endettés de la zone euro.

29 En 2008, les déficits courants en \% du PIB étaient de: - 17,1 \% (Grèce), - 14,4 \% (Portugal), - 11,0 \% (Espagne), - 6,7 \% (Irlande) et - 3,2 \% (Italie). Source: FMI. 
investis, réduisant ainsi l'excédent de la balance financière. Le déficit courant de ces pays s'est réduit lui aussi, de façon brutale et importante ${ }^{(30)}$, ce qui a représenté une baisse drastique de l'investissement et de la consommation. Si l'on suit le raisonnement à son terme, on devrait donc observer une baisse similaire de l'excédent courant et commercial de l'Allemagne. Or, ce n'est pas le cas. Comme le montre le graphique 1, l'excédent commercial allemand s'est replié sur 2008-2009 mais a vigoureusement repris depuis. Car un changement s'est produit dans la structure géographique du solde commercial. Depuis 2007-2008, le solde positif vis-à-vis du continent européen a fortement baissé, en relation avec la baisse importante de l'excédent allemand vis-à-vis de l'Espagne, du Portugal, de la Grèce, de l'Irlande et de l'Italie. Cette baisse a été compensée par une hausse de l'excédent vis-à-vis de l'Amérique et de l'Asie. Les pays concernés sont principalement les États-Unis, la Chine, la Corée du Sud et le Japon. On constate sur le graphique 5 un excédent commercial de l'Allemagne avec l'Asie pour la première fois en 2012.

Graphique 5: Balance commerciale de l'Allemagne par continent-zone, 1989-2013 (millions d'euros)

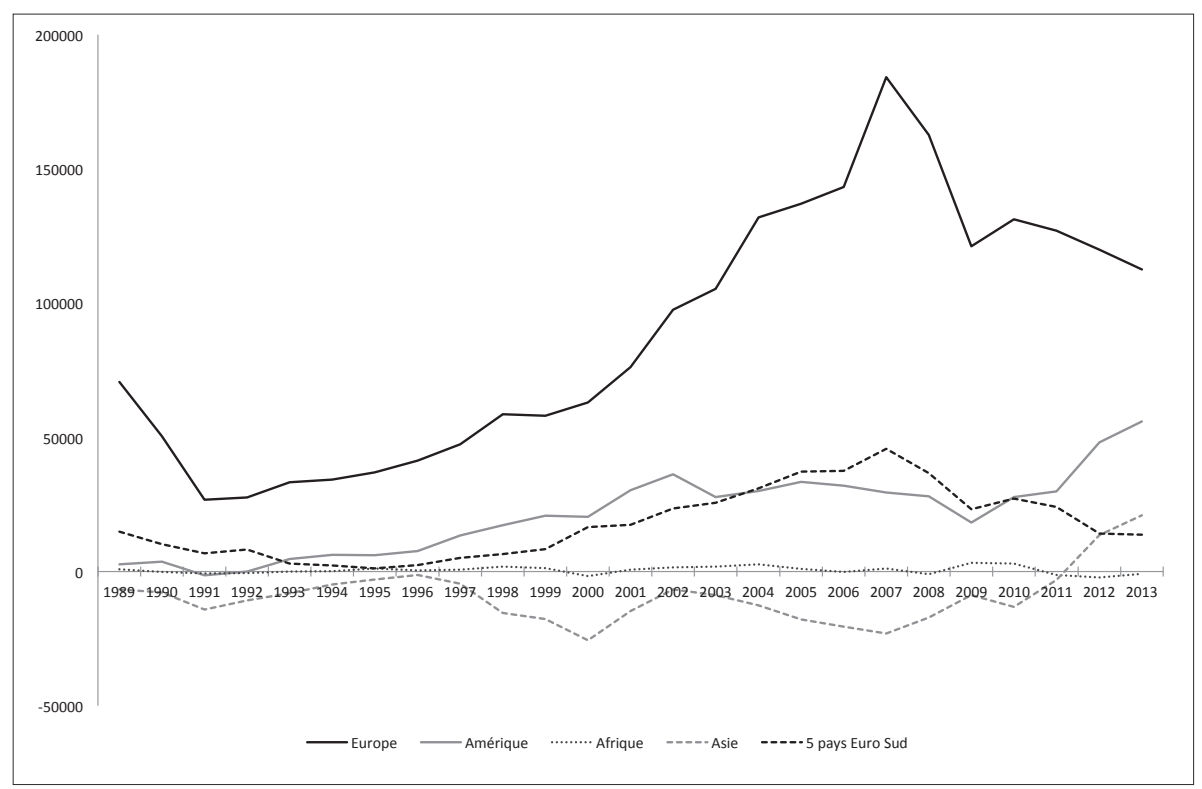

Source: D’après Statistisches Bundesamt, Fachserie 7 Reihe 1, Außenhandel. Zusammenfassende Übersichten für den Außenhandel (plusieurs années).

Qu'en est-il des excédents de capitaux allemands, aussi persistants que l'excédent commercial ? Le graphique 3 signale un changement sur 2006-2012. On voit que la Bundesbank devient le principal exportateur de capitaux (du type "Autres investissements »). Ainsi, ce ne sont plus des capitaux privés qui sont exportés d'Allemagne mais des capitaux publics. Ce phénomène met l'accent sur une des conséquences de l'appartenance à

30 En 2012, les déficits courants en \% du PIB sont de : - 3,5 \% (Grèce), - 1,6 \% (Portugal), - 1,1 \% (Espagne), - 4,8\% (Irlande) et - 0,8\% (Italie). Source: FMI. 
une union monétaire. Les banques centrales des pays membres n'ont plus à utiliser les réserves de change pour équilibrer la balance des paiements, comme c'est le cas dans un système de changes fixes ${ }^{(31)}$. Les réserves de change sont remplacées par des comptes des banques centrales des pays membres auprès de la banque centrale de l'union monétaire. Les banques centrales des pays excédentaires accumulent des positions créditrices sur les banques centrales des pays débiteurs, positions qui résultent des opérations entre résidents des pays de la zone monétaire ${ }^{(32)}$. Au sein de l'Union monétaire européenne, le système porte le nom de TARGET ${ }^{(33)}$. Il a été mis en place dès 1999 en même temps que l'introduction matérielle de l'euro afin d'aider à la mise en ouvre de la politique monétaire européenne et de faciliter les paiements interbancaires au sein de l'UE. Ce système, qui n’a pas une grande visibilité aux yeux du public, provoque un débat très pointu entre économistes sur les risques qu'il peut faire courir à la stabilité de la zone euro. En effet, il offre des facilités de crédit illimitées aux banques qui permettent le financement de déficits courants dans des proportions théoriquement illimitées ${ }^{(34)}$.

\section{Conclusion}

Cette analyse est partie d'une interrogation sur l'excédent commercial de l'Allemagne. Celui-ci est vivement critiqué de nos jours par ses partenaires européens en raison des dangers qu'il fait courir à l'équilibre de l'économie européenne. On l'attribue à la politique salariale du gouvernement allemand et à un manque de "bonne volonté » des Allemands qui n'auraient qu'à consommer plus et épargner moins pour que disparaissent les risques de crise. Ces critiques négligent un changement radical dans l'évolution de l'excédent commercial depuis 2000 qui a conduit au solde le plus élevé de toute l'histoire de l'Allemagne en 2013. Un tel changement ne peut être expliqué par des causes traditionnelles. L'explication avancée dans cet article appelle à considérer la balance des paiements allemande dans sa totalité et à prendre conscience qu'un changement majeur s'est également produit dans la balance financière depuis 2000 . Il renvoie à la création de l'Union monétaire européenne en 1999 qui a eu un impact immédiat sur les marchés de capitaux européens. L’UME a permis un accroissement massif des transactions financières et des transactions réelles. Cela s'est traduit par

31 Cf. plus haut les notions de base sur la balance des paiements. En cas de déficit courant et d'insuffisance des importations de capitaux privés pour couvrir ce déficit, la banque centrale va vendre des devises. Si la balance courante est en excédent et que les exportations nettes de capitaux privés sont inférieures à cet excédent, nécessairement les réserves de change de la banque centrale augmentent (devises obtenues par la vente de marchandises ou par les dépenses des touristes étrangers).

32 «A Greek importer, for example, might place an order with a German company. Payments to and from the accounts of the buyer and seller are channeled via central banks, so the German exporter's bank gets a credit with the Bundesbank, which in turn has a claim on the ECB. The Greek importer's bank owes its local central bank, leaving the Bank of Greece with a debit at the ECB », in : http://www.bloomberg.com/ news/2012-03-13/soaring-target2-imbalances-stoke-german-risk-angst-euro-credit.html.

33 Système de transfert express automatisé transeuropéen à règlement brut en temps réel.

34 Lire les points de vue divergents exposés dans Hans-Werner Sinn, Timo Wollmershaüser, Target Balances and the German Financial Account in Light of the European Balance-of-Payments Crisis, CESifo, WP 4051, décembre 2012, 21 p.; Jörg Bıвоw, The Euro Debt Crisis and Germany's Euro Trilemma, Levy Economics Institute of Bard College, WP 721, mai 2012, 43 p. 
une augmentation du même ordre des soldes de la balance des paiements au sein de la zone euro. L'Allemagne et les pays du sud de l'Europe ont été les pays les plus touchés.

L'analyse à moyen terme de la balance des paiements de l'Allemagne met donc en lumière un phénomène dont on ne peut prendre la mesure lorsqu'on privilégie une approche de court terme: les conséquences de l'euro sur l'évolution commerciale de l'Allemagne. L'union monétaire mise en place en 1999 a permis d'augmenter considérablement la création monétaire au sein de la zone euro, ce qui a accru dans des proportions également considérables - si l'on en juge par l'évolution de la balance commerciale allemande - les échanges de biens. L'ampleur du processus et la crise qu'il a provoquée laissent penser qu'il n'est pas parfaitement maîtrisé par les autorités monétaires européennes. Des mesures d'ajustement de la politique monétaire européenne paraissent inévitables, comme cela est à prévoir dans la mise en œuvre de toute nouvelle expérience. La présente étude permet ainsi de mieux comprendre l'interaction qui s'est mise en place entre la balance des paiements allemande et la construction monétaire européenne. Faut-il s'attendre à une baisse drastique de l'excédent commercial allemand ? Cela paraît peu vraisemblable à court terme. Il faudrait un arrêt brutal des flux de capitaux européens, ce qui n'est souhaité par personne. On peut avancer qu’à moyen-long terme, les échanges commerciaux allemands finiront par retrouver une plus grande dépendance vis-à-vis des déterminants traditionnels et que l'Allemagne sera alors confrontée à la dimension structurelle de ses échanges caractérisée par une forte concentration de ses exportations sur un nombre limité de produits ${ }^{(35)}$.

\title{
Résumé
}

L'excédent commercial massif de l'Allemagne est l'objet de nombreuses critiques. On l'attribue généralement à la politique du gouvernement allemand et aux habitudes de consommation des Allemands. Cet article propose une vision très différente basée sur l'observation de l'excédent allemand sur le long terme et relativement aux autres soldes de la balance des paiements. Il montre qu'un changement radical s'est produit en 2000 avec l'augmentation spectaculaire de l'excédent. Ce changement est à rapprocher de la création de l'Union monétaire européenne et de la crise de l'euro en 2008.

\begin{abstract}
The huge trade surplus of Germany is subjected to many criticisms and is usually ascribed to German politics and consumer habits. This article provides a very different view by considering the German trade surplus over the long run and relative to other accounts of the balance of payments. It shows a radical change since 2000 when a spectacular increase in the trade surplus occurred that appears to be linked to the creation of the European monetary union and the Euro crisis in 2008.
\end{abstract}

